\title{
Effects of Religious vs. Standard Cognitive Behavioral Therapy on Therapeutic Alliance: A Randomized Clinical Trial
}

Harold G. Koenig, ${ }^{1,2,3,4}$ Michelle J. Pearce, ${ }^{1,4,5}$ Bruce Nelson, ${ }^{6}$ Sally F. Shaw ${ }^{6}$ Clive J.

Robins, ${ }^{1,7}$ Noha Daher,${ }^{8,9}$ Harvey Jay Cohen,,${ }^{2,410}$ and Michael B. King ${ }^{11}$

${ }^{1}$ Department of Psychiatry and Behavioral Sciences, Duke University Medical Center, Durham, NC

${ }^{2}$ Department of Medicine, Duke University Medical Center, Durham, NC

${ }^{3}$ Department of Medicine, King Abdulaziz University, Jeddah, Saudi Arabia

${ }^{4}$ Center for Spirituality, Theology and Health, Duke University, Durham, NC

${ }^{5}$ School of Medicine, University of Maryland, Baltimore, MD

${ }^{6}$ Department of Research, Glendale Adventist Medical Center, Glendale, CA

${ }^{7}$ Department of Psychology and Neuroscience, Duke University Medical Center, Durham, NC

${ }^{8}$ Epidemiology, Biostatistics, and Population Medicine, School of Public Health, Loma Linda University, Loma Linda, CA

${ }^{9}$ Allied Health Studies, School of Allied Health Professions, Loma Linda University, Loma Linda, CA

${ }^{10}$ Center for the Study of Aging and Human Development, Duke University Medical Center, Durham, NC

${ }^{11}$ Division of Psychiatry, Faculty of Brain Sciences, University College, London, United Kingdom

Word count: 4,302 (text only)

*Corresponding Author: Harold G. Koenig, M.D., Box 3400 Duke University Medical Center, Durham, NC 27710. E-mail: Harold.Koenig@ duke.edu. Phone 919-681-6633; FAX 919-4713624.

Funding support provided by the John Templeton Foundation 


\title{
Effects of Religious vs. Standard Cognitive Behavioral Therapy on Therapeutic Alliance: A Randomized Clinical Trial
}

\begin{abstract}
BACKGROUND: Treatments that integrate religious clients' beliefs into therapy may enhance the therapeutic alliance (TA) in religious clients.

OBJECTIVE: Compare the effects of religiously-integrated CBT (RCBT) and standard CBT (SCBT) on TA in adults with major depression and chronic medical illness.

METHOD: Multi-site randomized controlled trial in 132 participants, of whom 108 (SCBT=53, RCBT=55) completed the Penn Helping Alliance Questionnaire (HAQ-II) at 4, 8, and 12 weeks. Trajectory of change in scores over time was compared between groups.

RESULTS: HAQ-II score at 4 weeks predicted a decline in depressive symptoms over time independent of treatment group $(\mathrm{B}=-0.06, \mathrm{SE}=0.02, \mathrm{p}=0.002, \mathrm{n}=108)$. There was a marginally significant difference in HAQ-II scores at 4 weeks that favored RCBT ( $\mathrm{p}=0.076)$; however, the mixed effects model indicated a significant group by time interaction that favored the SCBT group $(\mathrm{B}=1.84, \mathrm{SE}=0.90, \mathrm{df}=181, \mathrm{t}=2.04, \mathrm{p}=0.043, \mathrm{~d}=0.30)$.
\end{abstract}

CONCLUSIONS: While RCBT produces a marginally greater improvement in TA initially compared to SCBT, SCBT soon catches up.

Trial registration: ClinicalTrials.gov: NCT01208428

Source of funding: John Templeton Foundation

Word count: 158

Keywords: religious, spiritual, psychotherapy, CBT, major depression, chronic illness, therapeutic alliance 


\section{Background}

Meta-analytic and systematic reviews of research on "religion-accommodative" counseling and “spiritually modified" cognitive therapy have found that integrating clients' religious or spiritual belief into therapy is at least as effective as standard therapy, especially in the treatment of depression (McCullough, 1999; Hodge, 2006). These therapies support clients' religious or spiritual beliefs and values and utilize them to challenge dysfunctional cognitions and alter behaviors that generate depression. In a more recent review, Worthington and colleagues (2011) conducted a meta-analysis of 46 studies $(n=3,290)$ that examined outcomes from religious accommodative therapies and non-religious spiritual therapies, concluding that clients receiving religious accommodative therapies showed slightly greater improvement on psychological outcomes than those receiving secular psychotherapies $(\mathrm{d}=0.26)$. Might this have something to do with the development of the therapeutic alliance when a religiously-integrated therapy is conducted in religious clients?

Early in the $20^{\text {th }}$ century Freud emphasized the importance of the therapist displaying interest in and sympathetic understanding of the patient (Freud, 1912; 1913). Later in the 1950's, Carl Rogers stressed the importance of the therapist showing empathic concern and unconditional acceptance (“client-centered” approach) (Rogers, 1951; 1957). Many others since then have contributed to the idea that the relationship between therapist and patient is crucial to the success of therapy (Horvath \& Luborsky, 1993). One was Lester Luborsky, who in 1976 wrote about the "helping alliance" and its role in psychotherapy, building on his and others" seminal discovery that different therapies often produced similar results no matter what technique was used (Luborsky et al., 1975; Luborsky, 1976). He argued that a key therapeutic factor in all psychotherapies was the development of the therapeutic alliance (TA). To measure 
the TA for research and clinical applications, Luborsky developed and revised the Penn Helping Alliance Rating Questionnaire (HAQ-II) (Luborsky, 1976; Luborsky et al., 1983; Luborsky et al., 1996), one of many scales now used to assess this dimension.

The TA between therapist and client is now recognized as one of the most important factors that influences response to treatment in psychotherapy, especially treatment of depression (Luborsky et al., 1985; Krupnick et al., 1996; Horvath et al., 2011; Arnow et al., 2013). According to a meta-analysis of 90 independent studies examining the effects of the TA on psychotherapy outcomes, the overall weighted mean effect size (r) was 0.21 (range -0.06 to 0.89), which made up a little more than one-half of the entire effect size associated with treatment response (0.39) (Horvath, 2001; Norcross \& Hill, 2004). The effect size for the HAQII reported in that report was 0.27 (Cohen's $d=0.56$ ).

The TA is important for the success of cognitive behavioral therapy (CBT) in the treatment of depression (Krupnick et al., 1996; Waddington, 2002; Schnur \& Montgomery, 2010) and is key to a sustained treatment response (Weck et al., 2013). The importance of the TA has also been shown for CBT when administered by telephone for the treatment of depression in those with chronic medical illness (Applebaum et al., 2012), and delivering CBT by telephone has little effect on the TA (Stiles-Shields et al., 2014).

Does integrating religious belief into psychotherapy (CBT in particular) enhance the TA, given that $55 \%$ to $74 \%$ of clients would like to discuss religious/spiritual issues during therapy (Rose et al., 2001; Stanley et al., 2011)? Not surprising, many authors argue that integrating religious beliefs into therapy ought to improve the TA and the effectiveness of therapy. For example, Ross (1994) notes that, “disregard of the person's religious practice can prevent the development of a therapeutic alliance in much the same way that the alliance is threatened if the 
therapist is unmindful of the patient's culture" (p 8). Likewise, Asnaani and Hofmann (2012) say that integrating a client's religious beliefs into therapy is consistent with culturally responsive therapy, where the TA is established between therapist and client along cultural lines (one of which might be religious). In a qualitative study of 12 therapists involved in bereavement work with clients, Golsworthy and Coyle (2001) indicate that "An awareness of religious and spiritual perspectives and an openness to these in others was seen by participants as integral to therapy due to its influence on the development of the therapeutic relationship" (p $188)$.

Finally, in a naturalistic study of 220 clients and 51 therapists from Christian and secular counseling centers, Wade and colleagues (2007) examined the relationship between therapistclient congruence on religious commitment and the use of religious interventions on treatment outcomes. They found that similarity between clients' religious commitment and religious interventions was more important than therapist-client congruence in religious commitment. The matching of therapist and client religiosity did not predict either closer therapeutic relationships or better treatment outcomes. In contrast, the conducting of religious therapy in highly religious clients did enhance the therapeutic relationship and the outcomes of therapy.

Nevertheless, there is some question about what effect religiously-integrated psychotherapy and matching religious orientations of therapist with client might have on the TA. Referring to a qualitative study of 10 clients (8 religious) that explored the process of helpseeking and therapy in a secular setting, Post and Wade (2009) note that "Participants expressed mixed opinions about the importance of being matched with a therapist with similar religious/spiritual beliefs. Some felt that a mismatch allowed them to gain new insights, whereas 
others refrained from discussing religious/spiritual topics until they were certain that their therapist shared similar beliefs" (p 138).

To our knowledge, however, no randomized clinical trial (RCT) has actually measured the TA and compared changes in the TA over time between those receiving religiouslyintegrated therapy and standard secular therapy (at least for the treatment of depression). In their review of religion-accommodative psychotherapy outcome studies, Worthington and Sandage (2001) note that, "Unfortunately, these studies have tended to ignore relational or therapeutic alliance variables..." (p 475). In one of the few religiously-integrated psychotherapy studies that has measured the TA, Hook and colleagues (2013) conducted a naturalistic study (not an RCT) of couple's therapy in which they administered the Working Alliance Inventory-Short Form after sessions 1,4 and 8 . Both therapists and couples $(\mathrm{n}=68)$ were Christian and religious techniques were used in about one-half of the eight treatment sessions. Working alliance scores (range 428) were high although only slightly increased from session 1 (23.5, $\mathrm{SD}=3.3)$ to session 8 (25.0, $\mathrm{SD}=2.8$ ). What remains unknown, however, is whether matching religious patients with religiously-integrated therapy improves the TA during the course of therapy beyond that seen with standard therapy.

\section{Objectives}

In the present study, which compared religiously-integrated vs. standard CBT (RCBT vs. SCBT) in persons with major depression and chronic medical illness (all of whom were at least somewhat religious or spiritual) (Koenig et al., 2014), we examined the TA early on in therapy and changes over time in each treatment group. We hypothesized that (1) the TA early on in therapy would predict a significant decline in depressive symptoms independent of treatment 
group; (2) RCBT conducted by therapists experienced with integrating religious beliefs into therapy would be associated with a stronger TA early on in therapy than achieved by SCBT; (3) the TA would increase more during the course of therapy in those receiving RCBT than in those receiving SCBT; and (4) the effect would be particularly strong in clients who were Christian receiving Christian RCBT from Christian therapists, and in those who were highly religious.

\section{Methods}

\section{Trial design}

Design was a multi-site parallel randomized clinical trial conducted in two phases, each phase involving separate samples. During Phase I $(n=39)$, clients had a choice of receiving therapy by telephone, Skype, or instant messaging, with most choosing the telephone. Follow-up assessments for the Phase I sample were conducted at 4, 8 and 12 weeks from baseline. During Phase II (n=93) all treatment sessions were conducted by telephone and an additional 24-week follow-up was added (12 weeks after therapy ended). Overall, $94 \%$ of sessions were delivered by telephone, $5 \%$ by Skype (43 of 940), and 1\% by instant messaging (14 of 940). Screening, recruitment, randomization, interventions, and all assessments were essentially the same for Phase I and Phase II samples.

\section{Participants}

Individuals were recruited from two sites, one on the East coast (Durham, North Carolina) and the other on the West coast (Los Angeles County, California). Inclusion criteria were (1) age 1885; (2) one or more chronic medical illness of six months or longer; (3) religion or spirituality at least somewhat important; (4) a DSM-IV diagnosis of major depressive disorder (MINI 
Neuropsychiatric Inventory; Sheehan et al., 1998, was used to identify major depressive disorder and rule out mental disorders and suicidal thoughts that were exclusions); and (5) mild to moderately severe depressive symptoms (see below). Exclusion criteria were (1) significant cognitive impairment; (2) psychotherapy in the past two months; (3) psychotic disorder, alcohol or substance abuse, or post-traumatic stress disorder, within the past year, or any history of bipolar disorder; (4) active suicidal thoughts; (5) diagnosis of HIV/AIDS, autoimmune diseases, dementia, or endocrine disorders affecting stress hormone levels, or taking immunosuppressant drugs; (6) inability to communicate in written English; and (7) lack of remote access (i.e., a telephone, etc.). Interviewers screened potential participants by telephone for eligibility criteria and then arranged a visit when written informed consent was obtained and further screening was performed. Those who met eligibility criteria were enrolled and completed a baseline evaluation. All participants were told that treatment might or might not incorporate their religious beliefs and values. Duke University Medical Center institutional review board and Glendale Adventist Medical Center in LA County approved the study.

\section{Interventions}

The interventions consisted of ten 50-minute sessions administered over 12 weeks. SCBT was modeled after CBT for depression described by Aaron Beck and was manual-based (Beck et al., 1979). Both treatment groups addressed the cognitive distortions that persons with chronic illness often have, including issues regarding worthlessness, persistent disability, etc. Whenever religious issues came up during therapy, SCBT therapists redirected clients to more secular ways of approaching the issue. RCBT was specific to the religion of the client and was also manualbased. A Christian RCBT manual (Pearce et al., 2014) was first developed from the original 
SCBT manual above. University faculty from Jewish, Muslim, Hindu, and Buddhist religious traditions with extensive experience using CBT and integrating participants' religious beliefs into therapy adapted the prototype Christian RCBT manual to their respective religious traditions, resulting in five religion-specific RCBT manuals. Therapist and participant workbooks were also developed for each religious tradition that described homework material assigned in each session. When participants of a particular faith tradition entered the study, the faculty who had developed the RCBT manuals and workbooks helped supervise the therapy. In RCBT, clients were taught to use their religious teachings to replace negative and inaccurate thoughts with positive principles found in scripture, and included behaviors such as scripture memorization, contemplative prayer, and engagement in their faith community.

Both SCBT and RCBT integrated spiritual content into treatment sessions, with SCBT including mindfulness-based meditation and a focus on forgiveness, gratefulness, altruistic behaviors, and engagement in social community activities. Both interventions adapted CBT to the kinds of problems that those with chronic illness face (physical symptoms, disability, loss of control, etc.). Thus, the interventions were identical in all respects except that RCBT focused from the start of therapy on clients' religious beliefs and integrated those beliefs into the therapy, using them to alter depression generating cognitions and behaviors, whereas SCBT did not. The goal was to determine if utilizing the religious beliefs of clients in therapy (religious beliefs in particular) would help to strengthen the therapeutic alliance.

\section{Therapists}

Eight master's level therapists administered the interventions. The four conventional therapists delivering SCBT (two in NC and two in CA) were experienced cognitive therapists, but had no 
experience integrating religious beliefs into therapy. The four therapists who delivered RCBT (two in NC and two in CA) were Christian and experienced with both CBT and integrating religious beliefs into therapy. SCBT and RCBT therapists were trained for the trial and supervised by Duke Faculty skilled in SCBT (Robins) or in both SCBT and RCBT (Pearce). Therapists received onsite training first together as a group and then separately with their respective supervisors. Regular supervision by telephone was continued throughout the trial for all therapists. Christian therapists conducted the therapy adapted for other religions, although

they were supervised by clinicians who were of that religion and experienced administering CBT from the perspective of that religious tradition. To qualify as a study therapist, a score of 40 or higher was required on the Cognitive Therapy Rating Scale (CTRS) (Young \& Beck, 1980; Vallis et al., 1986).

An adapted version of the Adherence Rating Scale (ARS) was used to assess therapist adherence to the treatment manuals and session structure, as well as quality of the relationship, therapist competence, and therapist flexibility (Waltz et al., 1993). Sessions were tape recorded, transcribed, and rated by trained and supervised raters who were otherwise not directly involved in the study. Of these transcripts, the initial one-third was of consecutive sessions at the beginning of the study, whereas the remaining two-thirds were randomly selected from the other treatment sessions throughout the trial.

\section{Primary Outcome}

TA was measured using the 19-item revised Penn Helping Alliance Questionnaire (HAQII) (Luborsky et al., 1996), one of the most widely used measures in the field (Summers \& Barber, 2003) that has one of the highest correlations with outcomes for CBT (Fenton et al., 
2001). Examples of items are "I feel the therapist understands me" and "I like the therapist as a person." Each item is rated on a 6-point scale from 1 to 6 ("I feel strongly it is not true" to "I feel strongly it is true"), and the full scale has a range of 19 to 114 . The following studies provide examples of the usual scores obtained on the HAQ-II and changes in score during therapy. In the original report on the psychometric properties of the HAQ-II, Luborsky and colleagues (1996) examined development of the TA in 246 cocaine-dependent clients treated with psychotherapeutic interventions (including cognitive therapy). After the $2^{\text {nd }}$ treatment session, the mean HAQ-II score was 97.9 (SD 11.0) $(\mathrm{n}=201)$; after the $5^{\text {th }}$ session it was 99.9 (SD 10.5) $(\mathrm{n}=182)$; and after the $24^{\text {th }}$ session it was 100.7 (SD 11.8) ( $\left.\mathrm{n}=87\right)$. In a second study, Ruglass and colleagues (2012) examined the effects of CBT on outcomes in 223 women with PTSD and substance use disorders. After session 2, the average HAQ-II score was 99.5 and changed little by week 6 of therapy (99.8). In a study most like the present one, McEvoy and colleagues (2014) used the HAQ-II to assess TA in clients treated with CBT (70\% with major depression or dysthymia). After session 2, mean HAQ-II score was 98.3 (SD 9.5, n=84) and after session 12 (end of treatment) it was 103.5 (SD 9.7, n=45). Cronbach's alpha (standardized) for the HAQ-II in the present study ranged from 0.66 to 0.76 across the three time points, comparable to that reported by McEvoy and colleagues (alpha=0.70).

\section{Other Measures}

Depressive Symptoms. The 21-item Beck Depression Inventory (BDI) (Beck et al., 1961) assessed baseline severity of depressive symptoms necessary for study entry (10-40) and tracked depressive symptoms during the course of treatment. For "sadness," as an example of an item on the BDI, clients were asked to pick out one statement that best described how they felt 
during the past two weeks including presently: "I do not feel sad" (0), "I feel sad much of the time" (1), "I am sad all the time and I can't snap out of it" (2), or "I am so sad or unhappy that I can't stand it" (3). BDI scale range is 0-63, and the Cronbach's alpha in the present sample ranged from 0.83 to 0.94 across the five time points.

Physical Health. Physical functioning was assessed using the Duke Activity Status Index (DASI) (Hlatky et al., 1989), severity of medical illness by the Cumulative Illness Rating Scale (examining illness severity across 12 major organ systems) (Linn et al., 1968), and ICD-10 co-morbid medical illnesses by the Charlson Comorbidity Index (Charlson et al., 1987). The abbreviated Mini-Mental State Exam was used to diagnose significant cognitive impairment, defined as 13 or lower on a 0-18 point scale (Koenig, 1996).

Social Support. Social support was measured with the social interaction and subjective support subscales of the Duke Social Support Index (DSSI) (Landerman et al., 1989). This 11item version of the DSSI asks about time spent interacting with friends, neighbors, and family, both in person and on the telephone, and also assesses the subject's satisfaction with social interactions and support received. This measure has a score range of 11 to 33, and Cronbach's alpha in the present sample was 0.80 .

Religiosity. Religious involvement was assessed with single items measuring importance of religion ("not important" [1] to "very important" [4[), religious attendance ("never" [1] to "more than once/week" [6]), and private religious activity ("rarely or never" [1] to "more than once a day" [6]), and multi-item measures of spiritual experiences and intrinsic religiosity. Spiritual experiences were measured using the 16-item Daily Spiritual Experiences Scale (DSES) (Underwood \& Teresi, 2002). "I feel God's presence" is an example of an item on this scale, with possible responses ranging from "never or almost never" (1) to "many times a 
day" (6). Scale range is 16-94, and Cronbach's alpha in the present sample was 0.94. Intrinsic religiosity was measured using the 10-item Intrinsic Religiosity Scale (Hoge, 1972). "My religious beliefs are what really lie behind my whole approach to life" represents an item on this scale, with possible responses ranging from "definitely not true" (1) to "definitely true of me" (5). Scale range is 10-50, and Cronbach's alpha in the present sample was 0.87 .

\section{Sample Size}

The sample size was determined based on the BDI, the primary outcome in the parent study (Koenig et al., 2014). Our primary hypothesis in this report was that RCBT would be more effective than SCBT in maintaining a TA in religious clients. The power of detecting a treatment difference of 7 points on the HAQ-II (SD 14.5) or a medium treatment effect of 0.5 at an alpha of $5 \%$ (one-tailed test) with 55 in each treatment group was $83 \%$.

\section{Randomization and Blinding}

A clinical trials' coordinating center randomized participants to either RCBT or SCBT using a 4person block design to ensure approximately equal numbers in each treatment group. The coordinating center then connected participants with their respective therapists and monitored the treatment process and other aspects of the trial. Study interviewers who conducted the screening, baseline, and follow-up evaluations were blinded to treatment group, a blind that was carefully maintained throughout the study. Follow-up assessments were largely self-rated and completed with minimal assistance. 


\section{Statistical Methods}

Hypothesis 1: The TA early on in therapy would predict a significant decline in depressive symptoms independent of treatment group. Growth curve modeling using random intercept and slope (mixed effect regression models) examined the effect of the HAQ-II score on trajectory of change in BDI through 12 weeks (depression outcome). This method allowed for participants with data for at least one time point to be included in the analysis and allowed the retention of participants with missing data. The model included the fixed effects of group and HAQ-II score at week 4, time, time-squared, group by time interaction, and HAQ-II score at week 4 by time interaction.

Hypothesis 2: RCBT would be associated with a stronger TA early on in therapy than achieved by SCBT. Student's t-test was used to compare HAQ-II scores at 4, 8, and 12 weeks between treatment groups.

Hypothesis 3: In order to examine the trajectory of change in HAQ-II scores over time between treatment arms (the primary outcome), the fixed effects of group, time, and group by time interaction were included in a mixed model (time-squared was not significant). To address therapist effects in the model, an 8-level categorical variable was created to represent each of the 8 therapists in the study, and that variable was fit as a random effect in a 3-level model.

Hypothesis 4: The effect of RCBT on trajectory of change in HAQ-II scores over time would be particularly strong in clients who were Christian receiving Christian RCBT from Christian therapists, and in those who were highly religious. The model in hypothesis 3 above was repeated in Christian clients only and in those who were highly religious. To identify participants in the highly religious group, a religiosity measure was created by summing responses for religious attendance, private religious activity, religious importance, daily spiritual 
experiences, and intrinsic religiosity (standardized $\alpha=0.88$ ). Those scoring at least one standard deviation above the mean on this measure were defined as highly religious.

Given that screening, recruitment, randomization, interventions, and all assessments were essentially the same for Phase I and Phase II samples, we combined them for analysis. In order to test whether phase made a difference in outcome (hypothesis \#3), a moderator analysis was conducted by including phase, phase by group, phase by time, and phase by group by time.

Effect size (Cohen's d) was calculated from the t score and degrees of freedom (df) in each model. All statistical analyses were done using SAS (version 9.3; SAS Institute Inc., Cary, North Carolina). Significance level was set at $\mathrm{p}<0.05$ for the primary endpoint set at 12 weeks (and other effects reported in this paper as well).

\section{Results}

Of the 132 participants randomized to treatment group between June 15, 2011, and June 14, 2013, 108 completed the HAQ-II after 4 weeks, 94 after 8 weeks, and 89 after 12 weeks. As indicated in Figure 1, clients were not more likely to drop out in one condition vs. the other. Clients who dropped out did not have significantly higher levels of depression than those who completed at least five treatment sessions $(\mathrm{BDI}=22.3, \mathrm{SD}=9.1$, vs. $\mathrm{BDI}=25.6, \mathrm{SD}=7.9$, respectively, $\mathrm{df}=106, \mathrm{t}=1.61, \mathrm{p}=0.110$ ). However, dropouts did have a lower 4 -week TA scores compared to those who completed therapy $(\mathrm{TA}=85.6, \mathrm{SD}=16.8$, vs. $\mathrm{TA}=95.3, \mathrm{SD}=13.5$, respectively, $\mathrm{df}=106, \mathrm{t}=-2.67, \mathrm{p}=0.009)$. A comparison of baseline characteristics of the 108 participants who completed the 4-week HAQ-II by treatment group is presented in Table $\mathbf{1}$. There were no statistically significant differences between the groups on baseline characteristics, 
except on manual fidelity, where those in the SCBT group scored significantly higher on flexibility (see below).

\section{Treatment Fidelity}

Among clients who completed the HAQ-II, 82 transcripts (9\% of all sessions) were rated by outside CBT experts using an adapted version of the Adherence Rating Scale on the dimensions of session structure (range 0-15), therapeutic relationship (range 0-6), adherence to manual (range 0-8), therapist competence (range 1-4), and flexibility (range 1-4), with higher scores indicating greater adherence. Overall mean (SD) scores were 13.2 (2.0) for session structure, 5.4 (0.8) for therapeutic relationship, 6.8 (1.1) for adherence to manual, 3.2 (0.6) for competence, and 3.2 (0.6) for flexibility, with an average total score of 31.9 (4.2). SCBT and RCBT therapists were not statistically significantly different for each dimension and the total score, except that the SCBT therapists showed more flexibility than RCBT therapists (3.4 vs. 3.1, $\mathrm{df}=78, \mathrm{t}=2.30, \mathrm{p}=0.024)$.

\section{Therapeutic Alliance}

The average HAQ-II score for participants after 4 weeks of therapy was 93.7 (SD 14.5, range 49114). At the completion of therapy after 12 weeks, HAQ-II scores increased slightly to 97.6 (SD 13.5, range 49-114). The average change in HAQ-II score from $4^{\text {th }}$ to the $12^{\text {th }}$ week was 2.6 points $(\mathrm{SD}=9.1$, range $=-20.0$ to 31.0$)$. Although Christian therapists conducted the RCBT with non-Christian patients, there was no significant difference in TA at any time point between Christians and non-Christians in the RCBT group (results not shown). 


\section{Therapeutic Alliance and Treatment Outcome (Hypothesis 1)}

Across SCBT and RCBT groups, average BDI score dropped from 25.3 at baseline to 11.8 at the 12-week follow-up. As expected, initial TA affected depression outcomes independent of treatment group. The random coefficients regression models predicting BDI outcome indicated that the interaction between HAQ-II scores assessed early in therapy (week 4) and time predicted treatment response at both 12 weeks $(B=-0.06, \mathrm{SE} 0.02, \mathrm{df}=290, \mathrm{t}=-3.13, \mathrm{p}=0.002$, Cohen's $\mathrm{d}=0.37)$ and 24 weeks $(\mathrm{B}=-0.05, \mathrm{SE}=0.02, \mathrm{df}=353, \mathrm{t}=-3.49, \mathrm{p}<0.001$, Cohen's $\mathrm{d}=0.37)$. This indicates a small to medium effect size for TA on BDI outcome independent of treatment group both immediately after therapy ended and at 3 months afterwards.

\section{Treatment Group and Therapeutic Alliance (Hypotheses 2-4)}

TA appeared to be slightly higher in those receiving RCBT (vs. SCBT) after 4 weeks of therapy (96.1 vs. 91.2, F=-1.79, $\mathrm{p}=0.076$ ) (Table 2). However, this relative advantage for RCBT narrowed during the rest of therapy from 5.0 points after 4 weeks to 1.7 points after 8 weeks to 0.9 points after 12 weeks (see Figure 2). Thus, the mean difference in TA between SCBT and RCBT decreased by 3.7 points ( $\mathrm{SD}=9.0,95 \% \mathrm{CI}-0.06$ to $7.49, \mathrm{p}=0.053, \mathrm{n}=89$ ) during the course of therapy. Looking at individual items on the HAQ-II at the $4^{\text {th }}$ week, those receiving RCBT scored significantly higher than those receiving SCBT on item 5 ("I feel I am working together with the therapist in a joint effort") [5.38 (0.76) vs. 4.83 (1.28), t=2.7, p=0.008], item 15 ("The therapist and I have meaningful exchanges") [5.24 (1.04) vs. $4.73(1.26), t=2.3, p=0.026]$, and item 18 ("I believe the therapist likes me as a person") [5.12 (0.54) vs. 4.77 (1.01), t=2.2, $\mathrm{p}=0.031]$. By the $12^{\text {th }}$ week, however, no significant differences were found between treatment groups on any of the 19 HAQ-II items. 
The random coefficients regression model indicated a marginally significant main effect for group $(\mathrm{B}=-6.61, \mathrm{SE} 3.92, \mathrm{df}=181, \mathrm{t}=-1.69, \mathrm{p}=0.094, \mathrm{~d}=0.25)$ and a weak significant group by time interaction $(\mathrm{B}=1.84, \mathrm{SE}=0.90, \mathrm{df}=181, \mathrm{t}=2.04, \mathrm{p}=0.043, \mathrm{~d}=0.30)$ (Table 3). The latter suggests that the rate of TA improvement in the SCBT group was greater than the rate of improvement in the RCBT group (which is consistent with Figure 2). Results were similar for highly religious clients and for those receiving at least 5 therapy sessions (per-protocol).

In order to ensure that combining participants in phase I and II for the overall analysis did not significantly impact outcomes, a moderator analysis was performed. There was no statistically significant difference in the treatment effects.

\section{Discussion}

As expected, a higher therapeutic alliance between therapist and client early on in therapy significantly predicted treatment outcome (depressive symptoms) independent of treatment group both during therapy and for at least three months after therapy ended. Consistent with our hypothesis, there was also a suggestion that the TA established early in therapy was higher for those receiving religious-integrated than standard CBT. While RCBT therapists took somewhat less time to establish a good TA compared to SCBT therapists, the latter soon caught up. These findings are consistent with research showing that the TA is established and predictive of outcomes regardless of type of therapy (Wampold et al., 1997) (although, admittedly, the latter is controversial [see DeRubeis et al., 2005]). Nevertheless, the finding that those receiving RCBT did not experience an increase in TA over time relative to those receiving SCBT (and if anything, the opposite) is somewhat contrary to our expectations. This was also true for 
Christians receiving Christian CBT by Christian therapists, as well as for highly religious participants (all of whom were Christian).

\section{Interpretation}

How do we make sense of such findings that seem to run counter to our $2^{\text {nd }}$ hypothesis and the findings of at least one naturalistic study (Wade et al., 2007)? Why didn't RCBT that integrated clients' religious beliefs into therapy show a greater improvement in the TA over time compared to SCBT in these religious clients? Could this reflect loss of high expectation in the RCBT arm and/or getting over initial disappointment in the SCBT arm? Could this result from a ceiling effect, i.e., those receiving RCBT had less room to improve after the $4^{\text {th }}$ session compared to the SCBT group? Past research has shown that the HAQ-II plateaus around 100 (Luborsky e al., 1996; Ruglass et al. 2012; McEvoy et al., 2014).

Although matching the type of therapy with the religion and religiosity of the client to enhance the TA makes sense, this may not always be successful. In a study of 152 university students receiving services at a counseling center in a private Mormon university in Utah, Martinez and colleagues (2007) asked students which of the religious interventions they received were ineffective. Ineffective interventions reported by the $37 \%$ of participants who answered that question included scripture memorization, referral for a priesthood blessing, prayer between therapist and client, therapist encouraging client confession, therapist prayer, and therapist blessings. Negative consequences of such religious interventions included an increase in anxiety or guilt and feelings that therapy was becoming mixed up with religious doctrines. Although none of these components (except memorizing scripture) were included in our version of RCBT, a similar dynamic may have been present for some subjects here. 
There is also some uncertainty about the benefits of initiating a focus on clients' religious beliefs, as our RCBT therapists did. Both Knox and colleagues (2005) and Martinez and colleagues (2007) reported that clients preferred therapists to follow their lead with regard to addressing religious/spiritual issues in therapy. Addressing such issues worked best when clients themselves brought them up gradually during the course of therapy. When therapists initiated such discussions, this made some clients feel that therapists were imposing their religious beliefs on them. This was particularly true in the Knox et al study that involved spiritually diverse clients who did not identify with a particular religious group. In the present study, while religion/spirituality was at least somewhat important for all participants, religion was "very important" for only $47 \%$ of our sample compared to $56 \%$ of the U.S. population who indicate this (Pew Forum, 2007). For over one-third of participants (34\%), religion was either only somewhat important or not important at all, and half of these individuals (49\%) got RCBT. Perhaps getting free therapy in addition to modest remuneration may have drawn in participants for whom religion was not that important.

According to Ackerman and Hilsenroth (2003), the factors most important to the development of the TA are the therapist's flexibility, honesty, respect, trustworthiness, confidence, warmth, interest and openness. Peteet (2013) stressed that the TA is formed as the patient begins to feel understood, respected and cared for by the therapist who listens for the meaning of the patient's experiences with empathy, openness and wisdom. In fact, a metaanalysis of 31 studies that used religious or spiritual adaptations to psychotherapy concluded that the effectiveness of religious interventions in religious clients was due to general factors affecting the TA as much as to the nature of the therapy itself (Smith et al., 2007). 
The above reports could help to explain why the increase in TA after session 4 in those receiving RCBT did not exceed that in those receiving SCBT. Note that the amount of material RCBT therapists had to cover during treatment sessions was $30 \%$ more than standard CBT therapists had to cover during the allotted time. Not only did RCBT therapists have to learn a new way of integrating religious beliefs into therapy that followed our RCBT manual, they also had the additional RCBT material to cover that SCBT therapists didn't have to deal with. Having more material to cover in the same time and knowing that they would be rated on adherence to the manual, RCBT therapists may have had less time to go "off script" in order to listen to and empathize with clients. This situation would result in RCBT therapists being less flexible during sessions, which is exactly what we found when CBT raters examined the session transcripts. Thus, the findings here may be partly a consequence of the technical aspects of the study.

\section{Limitations and Generalizability}

The findings here should be interpreted and generalized with caution given the uniqueness of our RCBT intervention, delivery of therapy by telephone, the relatively small sample of participants with high religiosity, and relatively low religiosity of participants overall compared to the U.S. population in general. Given the initial differences in TA between SCBT and RCBT, especially the relatively low TA for SCBT (91.2), regression to the mean may be another explanation for an increase in TA over time for SCBT relative to RCBT (although is unlikely since the initial TA wasn't measured until 4 weeks of therapy). Finally, as noted above, comparing the TA that develops during a carefully controlled and monitored RCT may not be the same as the TA that develops naturally during clinical practice. 


\section{Conclusions}

We assessed the TA using a psychometrically valid and commonly used measure in a RCT examining the effects of religiously-integrated CBT compared to standard CBT among clients who were at least somewhat religious or spiritual. TA predicted treatment outcome independent of treatment group, an effect that was small to moderate in size. The TA that developed between therapist and client was initially somewhat stronger among those receiving RCBT than those receiving SCBT, suggesting that clients in the RCBT condition formed a stronger alliance with their therapists early in therapy (i.e., sessions 1-4). That difference, however, rapidly narrowed during the course of the therapy as clients in the SCBT condition caught up. Future research will be needed to determine whether this finding is unique to the present study, the RCBT intervention used here, and/or the constraints involved in a RCT.

Trial registration: ClinicalTrials.gov: NCT01208428

Protocol: full protocol can be accessed at:

http://www.spiritualityandhealth.duke.edu/index.php/religious-cbt-study/study-design Funding: The study sponsor (John Templeton Foundation) had no role in the study design, collection or analysis of data, the interpretation of data, or in the writing of this report. 
Figure 1. Participant flow

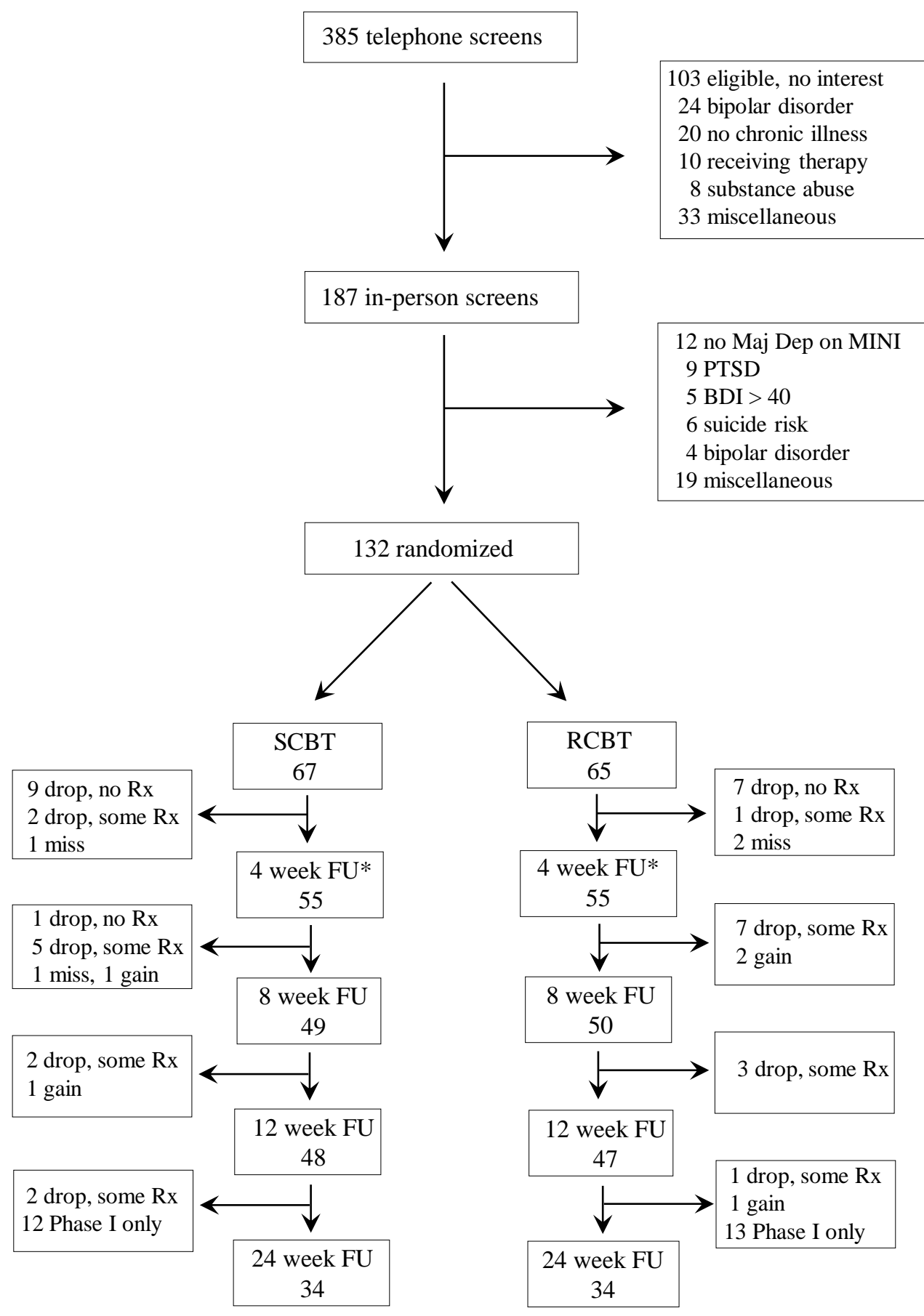

SCBT $=$ Standard Cognitive Behavioral Psychotherapy. RCBT=Religiously integrated CBT * 108 of 110 clients at 4-week follow-up completed the Penn Helping Alliance Questionnaire (HAQ-II), 53 in the SCBT group and 55 in the RCBT group 
Figure 2. Effects of standard vs. religious CBT on therapeutic alliance (mean, standard error)

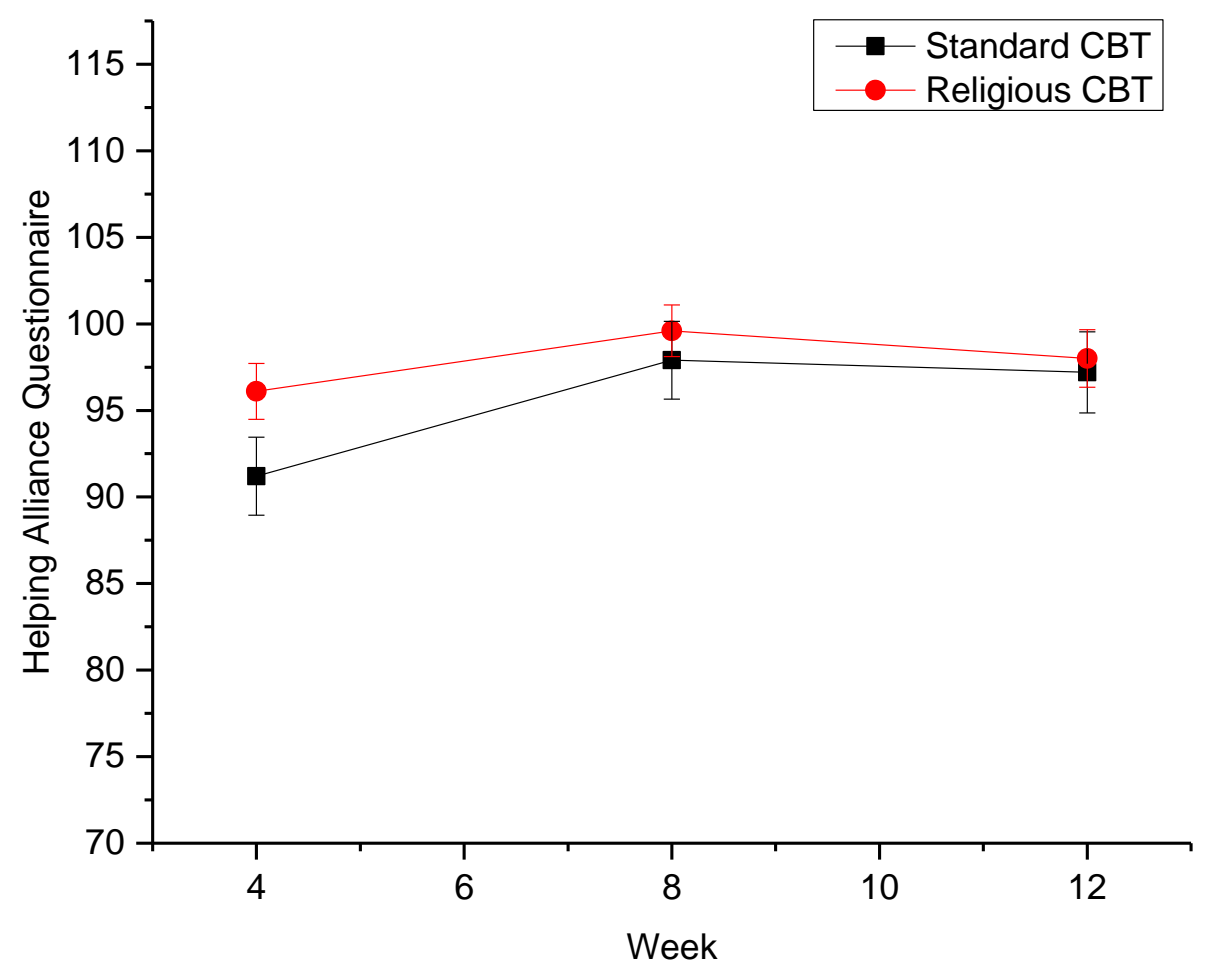


Table 1: Baseline characteristics by treatment group $(n=108)$

Demographics

Gender (female), \% (n)

$$
\frac{\text { SCBT }}{(n=53)}
$$

$\underline{\mathrm{RCBT}}$

$(n=53)$

$(\mathrm{n}=55)$

Age (years), mean (SD)

$\begin{array}{ll}64.2(34) & 74.6(41) \\ 52.9(14.0) & 51.1(13.3) \\ 58.5(31) & 49.1(27) \\ 15.3(3.4) & 15.1(3.6) \\ 52.8(28) & 36.4(20)\end{array}$

$\underline{\text { Religious characteristics }}$

Christian affiliation, \% (n)

Importance (very), \% (n)

$90.6(48)$

$80.0(44)$

$43.4(23)$

$50.9(28)$

$41.5(22)$

$43.6(24)$

Attendance (=/> weekly), \% (n)

$37.7(20)$

$34.6(19)$

$34.0(8.7)$

$35.2(8.8)$

Intrinsic (IRS), mean (SD)

$56.5(16.8)$

$58.0(15.8)$

Physical illness severity

Phys function (DASI), mean (SD)

$28.8(5.9)$

$28.4(5.9)$

$6.4(4.3)$

$7.0(5.6)$

$3.0(2.9)$

$2.5(2.3)$

Comorbidity $(\mathrm{CCI})$, mean (SD)

Depression

Symptoms (BDI), mean (SD)

$25.5(9.0)$

$24.6(7.2)$

$71.7(38)$

$74.6(41)$

$75.5(40)$

$76.4(42)$

Recurrent depression (>2), \% (n)

Study design

Manual fidelity, mean (SD) ${ }^{1}$

$\begin{array}{lrr}\text { Session structure } & 13.0(2.3) & 13.3(1.6) \\ \text { Therapeutic relationship } & 5.4(0.8) & 5.4(0.8) \\ \text { Adherence } & 6.8(1.1) & 6.9(1.0) \\ \text { Competence } & 3.3(0.6) & 3.1(0.6) \\ \text { Flexibility } & 3.4(0.6) & 3.1(0.7) \\ \text { Total score } & 32.0(4.5) & 31.7(3.9) \\ \text { Durham), \% (n) } & 45.3(24) & 45.5(25) \\ \text { (II), \% (n) } & 69.8(37) & 70.9(39) \\ \text { type, \% (n) } & & \\ \text { Christian } & ---- & 85.5(47) \\ \text { Non-Christian } & ---- & 14.6(8)\end{array}$

$\mathrm{SD}=$ standard deviation; IRS=Intrinsic Religiosity Scale; DSE=Daily Spiritual Experiences scale; CCI=Charlson Comorbidity Scale; DASI=Duke Activity Status Index; CIRS=Cumulative Illness Rating Scale; BDI=Beck Depression Inventory; CTS=Cognitive Rating Scale; CBT=Cognitive Behavioral Therapy; SCBT=standard CBT; RCBT=religiously-integrated CBT

${ }^{1} \mathrm{n}=41$ for SCBT group, $\mathrm{n}=41$ for RCBT group 
Table 2: Means (SD) of therapeutic alliance (HAQ-II) score by treatment group

$\begin{array}{llll}\text { SCBT } & \text { RCBT } & \text { t value } & \mathrm{p} \\ \text { Mean }(S D) & \text { Mean }(S D) & \end{array}$

\begin{tabular}{|c|c|c|c|c|}
\hline \multicolumn{5}{|l|}{ All Participants } \\
\hline HAQ-II at $8 \mathrm{wk}(\mathrm{n}=47 / 47)$ & $97.9(15.4)$ & $99.6(10.2)$ & -0.62 & .535 \\
\hline HAQ-II at 12 wk $(n=45 / 44)$ & $97.2(15.7)$ & $98.0(11.0)$ & -0.30 & .762 \\
\hline \multicolumn{5}{|l|}{ Participants (Christian) } \\
\hline HAQ-II at 4 wk $(n=48 / 44)$ & $91.3(15.5)$ & $95.7(12.6)$ & -1.47 & .145 \\
\hline HAQ-II at 8 wk $(n=42 / 36)$ & $97.7(15.9)$ & $99.9(10.3)$ & -0.74 & .460 \\
\hline HAQ-II at 12 wk $(n=40 / 34)$ & $96.7(16.0)$ & $98.5(10.0)$ & -0.60 & .551 \\
\hline \multicolumn{5}{|c|}{ Participants (highly religious) } \\
\hline HAQ-II at $4 \mathrm{wk}(\mathrm{n}=10 / 10)$ & $96.7(19.3)$ & $102.1(9.6)$ & -0.79 & .438 \\
\hline HAQ-II at 8 wk $(n=9 / 10)$ & $104.2(8.3)$ & $101.8(7.6)$ & 0.67 & .515 \\
\hline HAQ-II at 12 wk $(n=9 / 9)$ & $103.9(10.7)$ & $97.1(7.0)$ & 1.59 & .132 \\
\hline \multicolumn{5}{|l|}{ Per-protocol (all) } \\
\hline HAQ-II at $4 \mathrm{wk}(\mathrm{n}=45 / 45)$ & $92.8(15.3)$ & $97.8(11.0)$ & -1.76 & .082 \\
\hline HAQ-II at $8 \mathrm{wk}(\mathrm{n}=43 / 43)$ & $99.3(14.4)$ & $99.6(10.1)$ & -0.09 & .931 \\
\hline HAQ-II at $12 \mathrm{wk}(\mathrm{n}=43 / 41)$ & $97.3(16.0)$ & $97.6(11.1)$ & -0.09 & .929 \\
\hline \multicolumn{5}{|l|}{ Per-protocol (Christian) } \\
\hline HAQ-II at $4 \mathrm{wk}(\mathrm{n}=40 / 34)$ & $93.3(14.0)$ & $97.7(11.5)$ & -1.49 & .140 \\
\hline HAQ-II at $8 \mathrm{wk}(\mathrm{n}=38 / 32)$ & $99.2(14.8)$ & $99.9(10.2)$ & -0.21 & .833 \\
\hline HAQ-II at 12 wk $(n=38 / 31)$ & $96.8(16.4)$ & $98.0(9.9)$ & -0.35 & .725 \\
\hline
\end{tabular}

HAQ-II = Penn Helping Alliance Rating Questionnaire; SCBT=standard cognitive behavioral therapy, RCBT=religiously-integrated cognitive behavioral therapy, BDI=Beck Depression Inventory, wk=weeks, $\mathrm{M}=$ mean, $\mathrm{SD}=$ standard deviation, $\mathrm{p}=$ significance level 
Table 3: Effect of RCBT vs. SCBT on trajectory of change in therapeutic alliance (HAQ-II) from 4 weeks through 12 weeks during therapy

$$
\text { B coefficient Standard error } \quad p
$$

All Participants $(\mathrm{n}=108)$

Main effect of group

$-6.61$

3.92

.094

Group $\mathrm{x}$ time interaction

1.84

0.90

.043

Participants (Christian) (n=92)

Main effect of group

$-5.65$

4.09

.170

Group $\mathrm{x}$ time interaction

1.17

0.96

.226

Participants (highly religious) ${ }^{1}$

Main effect of group

$-11.18$

6.24

.082

Group $\mathrm{x}$ time interaction $(\mathrm{n}=20)$

Per-protocol (all) (n=90)

Main effect of group

Group $\mathrm{x}$ time interaction

Per-protocol (Christian) $(\mathrm{n}=74)$

Main effect of group

Group x time interaction

HAQ-II = Penn Helping Alliance Rating Questionnaire, SCBT=standard cognitive behavioral therapy, RCBT=religiously-integrated cognitive behavioral therapy

$B$ is unstandardized coefficient that represents the average difference between treatment groups ( $\mathrm{SCBT}=0, \mathrm{RCBT}=1$ ) from mixed effects growth curve models; $\mathrm{p}=$ significance level

${ }^{1}$ All 20 were Christian and received Christian CBT 


\section{References}

Ackerman, S. J., \& Hilsenroth, M. J. (2003). A review of therapist characteristics and techniques positively impacting the therapeutic alliance. Clinical Psychology Review 23:1-33.

Applebaum AJ, Duhamel KN, Winkel G, Rini C, Greene P, Mosher CE, Redd WH (2012). Therapeutic alliance in telephone-administered cognitive-behavioral therapy for hematopoietic stem cell transplant survivors. Journal of Consulting and Clinical Psychology 80:811-816

Arnow BA1, Steidtmann D, Blasey C, Manber R, Constantino MJ, Klein DN, Markowitz JC, Rothbaum BO, Thase ME, Fisher AJ, Kocsis JH (2013). The relationship between the therapeutic alliance and treatment outcome in two distinct psychotherapies for chronic depression. Journal of Consulting and Clinical Psychology 81(4):627-638.

Asnaani A, Hofmann SG (2012). Collaboration in culturally responsive therapy: establishing a strong therapeutic alliance across cultural lines. Journal of Clinical Psychology 68:187-197

Beck, A. T., Ward, C. H., Mendelson, M., Mock, J., Erbaugh, J. (1961) An inventory for measuring depression. Archives of General Psychiatry 4: 561-571

Beck AT, Rush J, Shaw BF, Emery G (1979). Cognitive Therapy of Depression. NY, NY: Guilford Press

Charlson ME, Pompei P, Ales KL, Mackenzie CR (1987). A new method of classifying prognostic comorbidity in longitudinal studies: development and validation. Journal of Chronic Disease 40(5):373-83

DeRubeis RJ, Brotman MA, Gibbons CJ (2005). A conceptual and methodological analysis of the nonspecific argument. Clinical Psychology: Science and Practice 12:174-183.

Fenton LR, Cecero JJ, Nich C, Frankforter TL, Carroll KM (2001). Perspective is everything: the predictive validity of six working alliance instruments. Journal of Psychotherapy Practice and Research 10, 262-268

Freud S (1912). The dynamics of transference. In J Starchey (ed), The Standard Edition of the Complete Psychological Works of Sigmund Freud, 1958. London: Hogarth Press, pp 99-108

Freud S (1913). On the beginning of treatment: further recommendations on the technique of psychoanalysis. In J Strachey (ed), The Standard Edition of the Complete Psychological Works of Sigmund Freud, 1958. London: Hogarth Press, pp 122-144

Golsworthy R, Coyle A (2001). Practitioners' accounts of religious and spiritual dimensions in bereavement therapy. Counselling Psychology Quarterly 14:183-202

Hlatky MA, Boineau RE, Higginbotham MB, Lee KL, Mark DB, Califf RM, Cobb FR, Pryor DB (1989). A brief self-administered questionnaire to determine functional capacity (The Duke Activity Status Index). American Journal of Cardiology 64:651-654 
Hodge DR (2006). Spiritually modified cognitive therapy: A review of the literature. Social Work 51:157-166

Hoge DR (1972). A validated intrinsic religious motivation scale. Journal for the Scientific Study of Religion 11:369-376

Hook JN, Worthington EL, Davis DE, Atkins DC (2013). Religion and couple therapy:

Description and preliminary outcome data. Psychology of Religion and Spirituality, Dec 23, epub ahead of print, doi: $10.1037 / \mathrm{a} 0035412$

Horvath AO, Luborsky L (1993). The role of the therapeutic alliance in psychotherapy. Journal of Consulting and Clinical Psychology 61:561-573

Horvath, AO (2001). The alliance. Psychotherapy: Theory, Research, Practice, Training, 38(4), 365-372.

Horvath AO, Del Re AC, Fluckinger C, Symonds D (2011). Alliance in individual psychotherapy. In JC Norcross (ed), Psychotherapy Relationships That Work: Evidence Based Responsiveness. NY, NY: Oxford University Press, pp 25-69

Knox S, Catlin L, Casper M, Schlosser LZ (2005). Addressing religion and spirituality in psychotherapy: Clients' perspectives. Psychotherapy Research 17:450-460

Koenig HG (1996). An abbreviated Mini-Mental State Exam for medically ill elders. Journal of the American Geriatrics Society 44:215-216

Koenig HG, Pearce MJ, Nelson B, Shaw SF, Robins CJ, Daher N, Cohen HJ, Berk LS, Belinger D, Pargament KI, Rosmarin DH, Vasegh S, Kristeller J, Juthani N, Nies D, King MB (2014). Religious vs. conventional cognitive-behavioral therapy for major depression in persons with chronic medical illness. Journal of Consulting and Clinical Psychology (in submission)

Krupnick JL, Sotsky SM, Simmens S, Moyer J, Elkin I, Watkins J, Pikonis PA (1996). The role of the therapeutic alliance in psychotherapy and pharmacotherapy outcome: Findings in the National Institute of Mental Health Treatment of Depression Collaborative Research Program. Journal of Consulting and Clinical Psychology 64:532-539

Landerman R, George LK, Campbell RT, Blazer DG (1989). Alternative models of the stress buffering hypothesis. American Journal of Community Psychology 17:625-642

Linn B, Linn M, Gurel L (1968). Cumulative Illness Rating Scale. Journal of the American Geriatrics Society 16:622-626

Luborsky L, Singer B, Luborsky L (1975). Comparative studies of psychotherapies: "Is it true that everybody has won and all must have prizes?" Archives of General Psychiatry 32:995-1008 
Luborsky L (1976). Helping alliances in psychotherapy. In JL Cleghhorn (ed), Successful Psychotherapy. NY, NY:Bruner/Mazel, pp 92-116

Luborsky L, L, Crits-Cristoph P, Alexander L, Margolis M, Cohen M (1983). Two helping alliance methods for predicting outcomes of psychotherapy: A counting signs vs. a global rating method. Journal of Nervous and Mental Disease 171:480-491

Luborsky L, Barber JP, Siqueland L, Johnson S, Najavits LM, Frank A, Daley D (1996). The revised Helping Alliance questionnaire (Haq-II): psychometric properties. Journal of Psychotherapy Practice and Research 5, 260-271

Luborsky L, McLellan AT, Woody Ge, O'brien CP, Auerbach A (1985). Therapist success and its determinants. Archives of General Psychiatry 42:602-611

Martinez JS, Smith TB, Barlow SH (2007). Spiritual interventions in psychotherapy:

Evaluations by highly religious clients. Journal of Clinical Psychology 63:943-960

McEvoy PM, Burgess MM, Nathan P (2014). The relationship between interpersonal problems, therapeutic alliance, and outcomes following group and individual cognitive behavior therapy. Journal of Affective Disorders 157: 25-32

McCollough ME (1999). Research on religion-accommodative counseling: Review and metaanalysis. Journal of Counseling Psychology 46:92-98

Norcross JC, Hill CE (2004). Empirically supported therapy relationships. Clinical Psychologist 57:19-24

Pearce MJ, Koenig HG, Robins CJ, Nelson B, Shaw SF, Cohen HJ, King MB. Religiouslyintegrated cognitive behavioral therapy: A new method of treatment for major depression in patients with chronic medical illness. Psychotherapy 2014, in press

Peteet J (2013). What is the place of clinicians' religious or spiritual commitments in psychotherapy? A virtues-based perspective. Journal of Religion and Health Dec 31. [Epub ahead of print]

Pew Forum (2007). U.S. religious landscape survey: beliefs and practices: Importance of religion. See website: http://religions.pewforum.org/maps

Post BC, Wade NG (2009). Religion and spirituality in psychotherapy: a practice-friendly review of research. Journal of Clinical Psychology 65:131-146

Rogers CR (1951). Client-Centered Therapy. Cambridge, MA: Riverside Press

Rogers CR (1957). The necessary and sufficient conditions of therapeutic personality change. Journal of Consulting and Clinical Psychology 22:95-103 
Rose, E. M., Westefeld, J. S., \& Ansley, T. N. (2001). Spiritual issues in counseling: Clients beliefs and preferences. Journal of Counseling Psychology 48, 61-71

Ross J (1994). Working with patients within their religious contexts: religion, spirituality, and the secular therapist. Journal of Systemic Therapies 13:7-15

Ruglass LM, Miele GM, Hien DA, Campbell ANC, Hu MC, Caldeira N, et al (2012). Helping alliance, retention, and treatment outcomes: a secondary analysis from the NIDA Clinical Trials Network Women and Trauma study. Substance Use \& Misuse 47:695-707

Schnur J, Montgomery G (2010). A systematic review of therapeutic alliance, group cohesion, empathy, and goal consensus/collaboration in psychotherapeutic interventions in cancer: Uncommon factors? Clinical Psychology Review 30:238-247

Sheehan BV, Lecrubier Y, Sheehan KH, et al: (1998). The Mini International Neuropsychiatric Interview (MINI): The development and validation of structured diagnostic psychiatric interview for DSM-IV and ICD-10. Journal of Clinical Psychiatry 59 (Suppl 20) :22-33

Smith TB, Bartz JD, Richards PS (2007). Outcomes of religious and spiritual adaptations to psychotherapy. A meta-analytic review. Psychotherapy Research 17:643-655

Stanley MA, Bush AL, Camp ME, Jameson JP, Phillips LL, Barber CR, Zeno D, Lomax JW, Cully JA (2011). Older adults' preferences for religion/spirituality in treatment of anxiety and depression. Aging and Mental Health, 15(3):334-343

Stiles-Shields C, Kwasny MJ, Cai X, Mohr DC (2014). Therapeutic alliance in face-to-face and telephone-administered cognitive behavioral therapy. Journal of Consulting and Clinical Psychology 82(2):349-354.

Summers RF, Barber JP (2003). Therapeutic alliance as a measurable psychotherapy skill. Academic Psychiatry 27, 160-165.

Tracey TJ, Kokotovic AM (1989). Factor structure of the Working Alliance Inventory. Psychological Assessment: 1:207-310

Underwood LG, \& Teresi JA (2002). The daily spiritual experiences scale: development, theoretical description, reliability, exploratory factor analysis, and preliminary construct validity using health-related data. Annals of Behavioral Medicine 24:22-33

Vallis TM, Shaw BG, Dobson KS (1986). The Cognitive Therapy Scale: psychometric properties. Journal of Consulting and Clinical Psychology 54, 381-385

Waddington L (2002). The therapy relationship in cognitive-behavioral therapy. Journal of Psychotherapy Practice and Research 1:207-210 
Wade NG, Worthington EL, Vogel DL (2007). Effectiveness of religiously tailored interventions in Christian therapy. Psychotherapy Research 17:91-105

Wampold BE, Mondin GW, Moody M, Stich F, Benson K, Ahn H (1997). A meta-analysis of outcome studies comparing bona fide psychotherapies: empirically, 'All must have prizes.' Psychological Bulletin 122:204-215.

Waltz J, Addis ME, Koerner K, Jacobson NS (1993). Testing the integrity of a psychotherapy protocol: assessment of adherence and competence. Journal of Consulting and Clinical Psychology 61, 620-630

Weck F, Rudari V, Hilling C, Hautzinger M, Heidenreich T, Schermelleh-Engel K, Stangier U (2013). Relapses in recurrent depression 1 year after maintenance cognitive-behavioral therapy: the role of therapist adherence, competence, and the therapeutic alliance. Psychiatry Research 210:140-145

Worthington EL, Sandage SJ (2001). Religion and spirituality. Psychotherapy: Theory, Research, Practice \& Training 38:473-478

Worthington EL, Hook JN, David DE, McDaniel MA (2011). Religion and spirituality. Journal of Clinical Psychology 67:204-214 